\title{
Advanced gastric adenocarcinoma mimicking a submucosal tumor
}

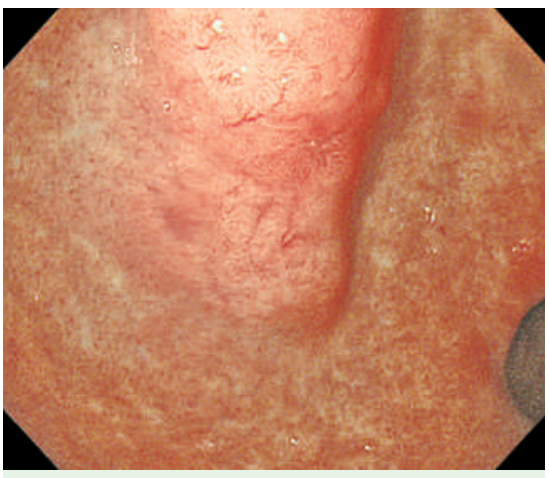

Figure 1 Initial endoscopy revealed a submucosal tumor covered with benign-appearing mucosa.

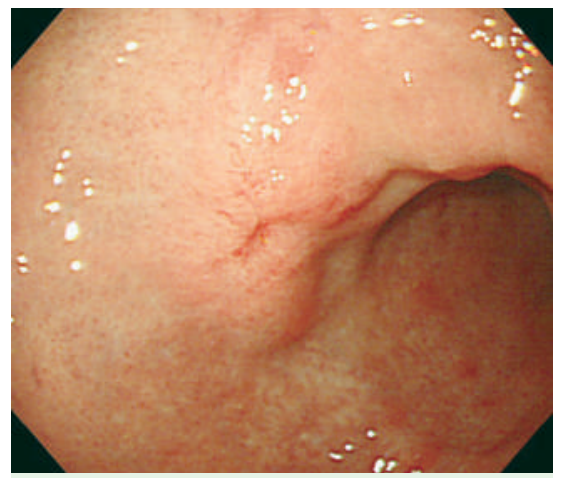

Figure 3 Follow-up endoscopy 13 months after the initial examination showed that the margin of the tumor had become more irregular, and the central depression with surrounding erythema had become deeper.

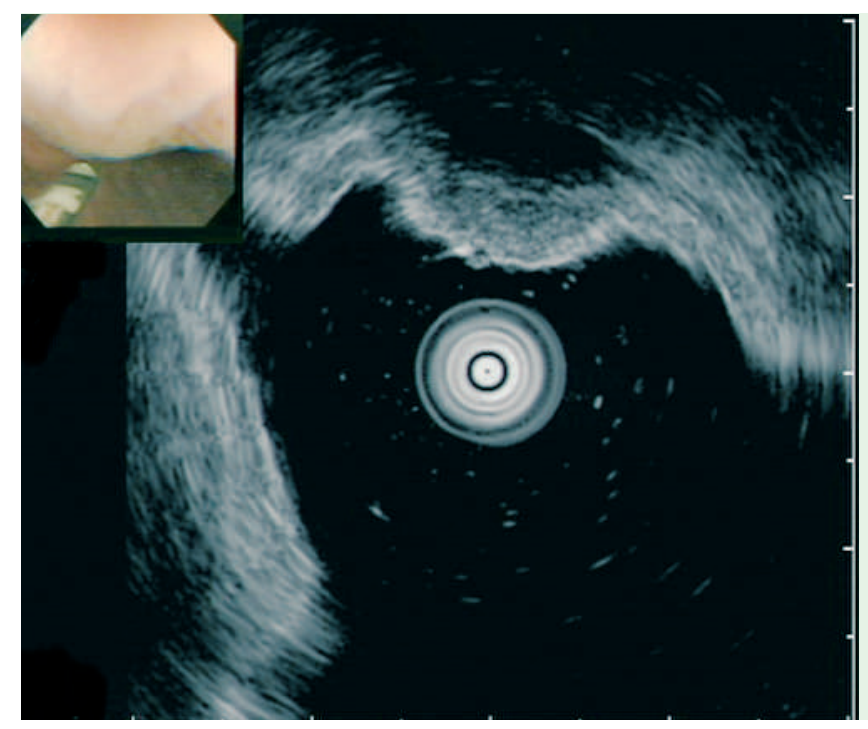

Figure 2 Endoscopic ultrasonography demonstrated a $13.0-\mathrm{mm}$ homogenous, circumscribed hypoechogenic lesion rising from the fourth sonographic layer.

A 63-year-old asymptomatic man was admitted for evaluation of a gastric lesion incidentally noted on upper gastrointestinal endoscopic examination during a routine health evaluation. At initial endoscopy, a 13-mm submucosal tumor covered with benign-appearing mucosa was found ( Figure 1). Endoscopic ultrasonography (EUS) demonstrated a 13.0$\mathrm{mm}$ homogenous, circumscribed hypoechogenic lesion rising from the fourth sonographic layer, and this lesion was thought from its morphology to be muscularis propria ( $\triangle$ Figure 2 ). Initial biopsy specimens were negative for any neoplas- tic degeneration. Follow-up endoscopy with mucosal biopsy was performed 13 months after the initial examination. This revealed that the margin of the tumor had become irregular, and the central erythema was more prominent (ه Figure 3). The specimens taken from the central depression revealed poorly differentiated adenocarcinoma. At laparotomy, serous surface invasion was strongly suspected on the gastric wall although no ascites or liver metastasis were present. Distal gastrectomy with regional lymph node dissection, cholecystectomy, and gastroduodenostomy were per-
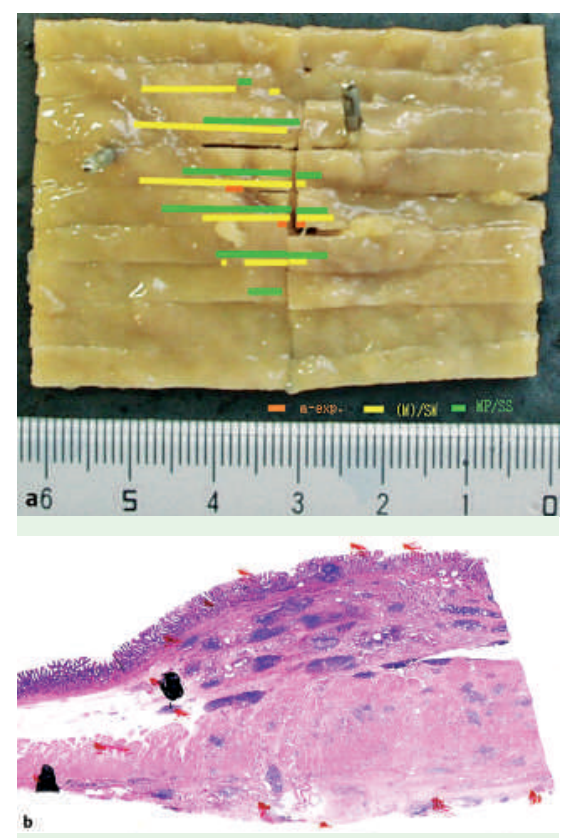

Figure 4 a Macroscopic view of the resected stomach, which measured $25 \times 20 \mathrm{~mm}$. The tumor extended to the muscularis propria/ subserosa (green line), submucosa (yellow line), and mucosa (orange line). b Photomicrograph of a cut section of the tumor demonstrating adenocarcinoma (H\&E staining, $\times 10)$.

formed. The histopathologic specimen was consistent with moderately differentiated adenocarcinoma, partially mixed with poorly differentiated adenocarcinoma, without focal lymph or vascular invasion. The tumor extended to the subserosa but did not involve the serosa ( Figures 4a,b). All dissected lymph nodes were free of tumor.

Although gastric cancer mimicking a submucosal tumor (GCSMT) is extremely rare [1] and it is difficult to obtain an adequate sample from the underlying lesion [2], the present case indicates that the finding of a small SMT with central erythema or granular changes and hypoechogenicity as defined by endoscopy and EUS should raise a suspicion of GCSMT. Consistently, previous reports have shown that GCSMTs were $33 \mathrm{~mm}$ or less in diameter at diagnosis, and all had central irregular erythematous or granular mucosa changes [3]. If the diagnosis is uncertain, the use of aggressive techniques instead of EUS alone, including EUS-guided biopsy, diagnostic endoscopic mucosal resection, possibly with surgical resection, should be advocated [4], and close follow-up is recommended.

Endoscopy_UCTN_Code_CCL_1AB_2AD_3AB

Endoscopy_UCTN_Code_CCL_1AF_2AD 


\section{F. Teraishi, F. Uno, S. Kagawa,}

T. Fujiwara, A. Gouchi, N. Tanaka

Department of Surgical Oncology,

Division of Surgery, Okayama University

Graduate School of Medicine,

Dentistry and Pharmaceutical Sciences,

Okayama, Japan

\section{References}

1 Tio TL, Tytgat GNJ, Jager FCA. Endoscopic ultrasonography for the evaluation of smooth muscle tumors in the upper gastrointestinal tract: an experience with 42 cases. Gastrointest Endosc 1990; 36: 342 - 350
2 Rösch T, Lorenz R, Dancygier H et al. Endosonographic diagnosis of submucosal upper gastrointestinal tract tumors. Scand J Gastroenterol 1992; 27: 1 -8

3 Fujiyoshi A, Kawamura M, Ishitsuka S. Gastric adenocarcinoma mimicking a submucosal tumor: case report. Gastrointest Endosc 2003; 58: 633-635

4 Wiersema MJ, Wiersema LM, Khusro Q et al. Combined endosonography and fine-needle aspiration cytology in the evaluation of gastrointestinal lesions. Gastrointest Endosc 1994; 40: 199-206
Bibliography

DOI $10.1055 / \mathrm{s}-2007-966403$

Endoscopy 2007; 39: E191 - E192

(c) Georg Thieme Verlag KG Stuttgart · New York . ISSN 0013-726X

\section{Corresponding author}

\section{F. Teraishi, MD}

Department of Surgical Oncology

Division of Surgery

Okayama University Graduate School of Medicine,

Dentistry and Pharmaceutical Sciences

Okayama 700-8558

Japan

Fax: +81-86-2217884

f-tera@yf7.so-net.ne.jp 